\title{
MODELAGEM DE TRÁFEGO FRACTAL UTILIZANDO PROCESSOS ENVELOPE
}

\author{
Flávio de M. Pereira, Nelson L. S. da Fonseca e Dalton S. Arantes
}

\begin{abstract}
Resumo - Neste artigo, propõe-se o uso de um processo envelope denominado Processo de Chegada com Limitante Fracionário (Fractional Bounded Arrival Process, FBAP) para a representação de tráfego auto-similar. A análise de sistemas de filas para o tráfego FBAP é desenvolvida, e limitantes para o tamanho da fila (backlog) e para o atraso são apresentados. O policiamento de tráfego FBAP também é estudado.Os resultados são estendidos para o caso do tráfego multifractal, para o qual se apresenta um processo envelope denominado Processo de Chegada com Limitante Multifractal (Multifractal Bounded Arrival Process, MFBAP). Tanto o FBAP como o MFBAP são validados utilizando traços de tráfego real, sendo comprovado que são capazes de representar adequadamente o tráfego com características fractais.
\end{abstract}

Palavras-chave: Análise de desempenho de redes, engenharia de tráfego. Internet, redes de comunicações banda larga, tráfego multifractal, processos envelope.

\begin{abstract}
In this paper, an envelope process called Fractional Bounded Arrival Process (FBAP) is proposed for selfsimilar traffic representation. A queueing analysis for FBAP traffic is developed, and upper bounds for the backlog and for the delay are obtained. The policing of FBAP traffic is also investigated. Results are then extended to the multifractal traffic case, for which an envelope process called Multifractal Bounded Arrival Process (MFBAP) is proposed. The FBAP and the MFBAP models are validated against real traffic traces, and they are proved to provide an adequate representation for fractal traffic.
\end{abstract}

Keywords: Network performance analysis, traffic engineering, Internet, wideband communications networks, multifractal traffic, envelope processes.

\section{INTRODUÇÃO}

Estudos recentes indicam que o tráfego em diversos tipos de redes apresenta propriedades fractais $[1,2,3,4,5,6,7,8$, $9,10,11,12,13]$. Em outras palavras, as propriedades estatísticas do tráfego não variam em relação à escala de tempo de observação, não sendo possível apontar uma escala de tempo dominante $[2,3,6]$.

Flávio de M. Pereira e Dalton S. Arantes pertencem à Faculdade de Engenharia Elétrica e de Computação, Universidade Estadual de Campinas, Campinas, SP, Brasil. Nelson L. S. da Fonseca pertence ao Instituto de Computação, Universidade Estadual de Campinas, Campinas, SP, Brasil. E-mails: \{pereira, dalton\}@decom.fee.unicamp.br,nfonseca@ic.unicamp.br. Editor de Área responsável: Abraham Alcaim. Artigo submetido em 24/Nov/2003, revisado em 15/Abr/2004, 17/Jul/2004, aceito em 1.3/Ago/2004.
Vários trabalhos têm sido direcionados à modelagem do tráfego fractal [13]. A importância destes estudos advém dos efeitos nocivos deste tipo de tráfego no desempenho das redes, os quais não podem ser adequadamente analisados mediante o uso de modelos de tráfego tradicionais [13]. De fato, o tráfego fractal é caracterizado pela ocorrência de surtos de grande intensidade em várias escalas de tempo. Além disso, este tipo de tráfego apresenta dependência de longa duração, que leva à persistência temporal dos surtos de tráfego, ocasionando significativas perdas por transbordo nos nós de comutação [13].

Diversos autores argumentam que o conceito de autosimilaridade estatística pode ser utilizado para caracterizar 0 comportamento do tráfego fractal $[2,3,4,6]$. Considera-se que um processo $\mathbf{Z}(t)$ com incrementos estacionários é autosimilar se a relação

$$
\mathbf{Z}(t) \stackrel{d}{=} m^{-H} \mathbf{Z}(m t), m, t \geq 0
$$

é válida para algum $0<H<1$. O símbolo $\stackrel{d}{=}$ indica igualdade em distribuição.

Modelos auto-similares foram propostos para a representação do tráfego em redes [13]. Em particular, Norros [14] propôs um modelo denominado tráfego Browniano fracionário, que é definido como:

$$
\mathbf{A}(t)=\mu t+\sigma \mathbf{Z}(t), t \geq 0,
$$

onde $\mathbf{A}(t)$ representa o tráfego acumulado até um dado instante $t, \mu>0$ é a sua taxa média, $\sigma \geq 0$ é um parâmetro associado ao seu desvio-padrão e $\mathbf{Z}(t)$ é um processo do tipo movimento Browniano fracionário, com média zero, fator de auto-similaridade $0.5 \leq H<1$ e variância var $\mathbf{Z}(t)=|t|^{2 H}$. Estudos recentes indicam que o processo $\mathbf{A}(t)$ também pode ser utilizado para a modelagem de tráfego com distribuição marginal $\alpha$-estável, assumindo que $\mathbf{Z}(t)$ é um processo do tipo movimento estável $[15,16]$. A análise de um sistema de filas simples, alimentado por tráfego Browniano fracionário foi apresentada em [17, 14].

Cabe observar que o tratamento matemático dos processos auto-similares geralmente não é trivial. Uma opção é o uso de limitantes para o tráfego, denominados processos envelope. Neste caso, o tráfego é modelado por meio de uma função determinística, à qual se associa uma probabilidade de violação, i.e., a probabilidade de que o tráfego exceda esta função. Caso esta probabilidade seja nula, o envelope é dito determinístico. Um processo envelope pode ainda ser classificado como acumulativo, se o limitante é imposto ao tráfego acumulado até um determinado instante de tempo, ou incremental, se ele é imposto à quantidade de tráfego dentro de um intervalo de tempo.

Em [18], propôs-se o uso de um processo envelope para a modelagem de tráfego auto-similar. Este processo, de- 
nominado processo envelope do movimento Browniano fracionário, é definido como

$$
\widehat{A}(t)=\mu t+k \sigma t^{H}, t \geq 0 .
$$

Os parâmetros $\mu, \sigma$ e $H$ são equivalentes aos parâmetros do tráfego Browniano fracionário. O parâmetro $k$ determina a probabilidade de que o processo $\mathbf{A}(t)$ exceda o envelope $\widehat{A}(t)$ no instante $t$, i.e.,

$$
\mathbb{P}\{\mathbf{A}(t)>\widehat{A}(t)\}=\mathbb{P}\{\mathbf{Z}(\mathbf{1})>k\}=\bar{\Phi}(k),
$$

onde $\bar{\Phi}(\cdot)$ representa a função distribuição Gaussiana residual. A partir da definição do processo envelope do movimento Browniano fracionário, um mecanismo de policiamento de tráfego auto-similar, denominado algoritmo do Balde Furado Fractal, foi apresentado em [19].

Note que, por definição, o processo envelope do movimento Browniano fracionário assume que o tráfego tem distribuição marginal Gaussiana, o que nem sempre é verdadeiro $[16,15,20]$. Além disso, este processo não representa adequadamente os incrementos de tráfego, em particular quando são supostos estacionários. Neste caso, a probabilidade de violação é dada por

$$
\begin{aligned}
\mathbb{P}\{\mathbf{A}(t+\tau)-\mathbf{A}(t) & >\widehat{A}(t+\tau)-\widehat{A}(t)\} \\
& =\mathbb{P}\left\{\mathbf{Z}(1)>k \frac{(t+\tau)^{H}-t^{H}}{\tau^{H}}\right\},
\end{aligned}
$$

o que não é consistente com a suposição de estacionariedade dos incrementos.

No presente artigo, é apresentado um processo envelope para modelagem de tráfego auto-similar, que é denominado Processo de Chegada com Limitante Fracionário (Fractional Bounded Arrival Process, FBAP). O modelo FBAP é capaz de representar tanto o tráfego incremental como o tráfego acumulado, e generaliza o processo envelope do movimento Browniano fracionário pois não supõe qualquer distribuição marginal para o tráfego. A análise de um sistema de filas simples para o tráfego FBAP também é apresentada, sendo obtidos limitantes para o tamanho da fila (backlog) e para o retardo introduzido por um sistema de filas simples com taxa de serviço constante.

$\mathrm{O}$ presente trabalho trata também do policiamento de tráfego auto-similar. Em [19], foi proposto um algoritmo para policiamento de tráfego auto-similar denominado Balde Furado Fractal (Fractal Leaky Bucket, FLB). O algoritmo FLB é um mecanismo de policiamento baseado em janelas, que restringe o tráfego a um envelope cumulativo e determinístico, dado por

$$
\widehat{A}(t)=\mu t+\psi t^{H}, t \geq 0 .
$$

Para que se obtenha uma probabilidade desprezível de descarte de tráfego em conformidade, os valores dos parâmetros do algoritmo FLB devem ser iguais ao do processo envelope do movimento Browniano fracionário, onde $\psi$ é dado pelo produto $k \sigma$.

Em [21], demonstrou-se que o algoritmo FLB não é capaz de limitar os surtos de tráfego auto-similar, e por isso não oferece suporte a garantias de desempenho. Uma opção é o uso do algoritmo do Balde Furado tradicional para o policiamento. Esta abordagem é especialmente interessante dado que há, na literatura, diversos estudos de análise de desempenho de redes que assumem que o tráfego é policiado por este algoritmo. Além disso, o algoritmo do Balde Furado já tem sido utilizado em redes devido à sua robustez e baixo custo computacional. No presente trabalho, o uso do algoritmo do Balde Furado para policiamento de tráfego FBAP é analisado, sendo obtidas relações matemáticas entre os parâmetros do algoritmo e os parâmetros do modelo.

Embora o conceito de auto-similaridade seja, em muitos casos, capaz de representar adequadamente as propriedades fractais do tráfego de redes, estudos recentes indicam que modelos mais sofisticados são por vezes necessários $[6,11$, 12, 22]. De fato, o tráfego pode exibir propriedades fractais mais complexas, que se distingüem significativamente da auto-similaridade [22]. Nestes casos, recorre-se ao conceito de multifractais para a modelagem.

Existem múltiplas definições para o conceito de multifractais [6, 12, 22, 23]. Em [23], um processo $\mathbf{Z}(t)$ com incrementos estacionários é denominado multifractal se a seguinte relação é válida

$$
\mathbf{Z}(m t) \stackrel{d}{=} \mathbf{C}(m) \mathbf{Z}(t), m, t \geq 0,
$$

onde $\mathbf{Z}(t)$ e $\mathbf{C}(m)$ são funções aleatórias independentes.

$O$ uso de processos multifractais para modelagem de tráfego ainda é um tema recente. Em [11], Riedi e Véhel analisaram diversos traços de tráfego TCP, e demonstraram que eles satisfazem relações de caráter multifractal. Em [12], Feldmann et al. propuseram o uso de processos multiplicativos para a modelagem do tráfego em redes. Em [22]. Molnár et al. analisaram o comportamento assintótico do tamanho da fila em um sistema de filas simples, alimentado por tráfego multifractal. Um tutorial sobre modelagem de tráfego por meio de processos multifractais é apresentado em [24].

No presente artigo, propõe-se o uso de um processo envelope para modelagem de tráfego multifractal, que é denominado Processo de Chegada com Limitante Multifractal ( $M u l$ tifractal Bounded Arrival Process, MFBAP).

$O$ restante deste artigo é organizado da seguinte maneira. Na Seção 2, o modelo FBAP é introduzido. Na Seção 3, a análise de um sistema de filas alimentado por tráfego FBAP é desenvolvida, sendo obtidos limitantes para o retardo e o backlog sob taxa de serviço constante. Na Seção 4, o policiamento de tráfego FBAP por meio do algoritmo do Balde Furado é analisado. Na Seção 5, o modelo MFBAP é introduzido. Finalmente, a Seção 6 conclui o artigo.

\section{O PROCESSO DE CHEGADAS COM LIMITANTE FRACIONÁRIO}

Seja o tráfego acumulado até o instante $t$ dado por (2), onde $\mathbf{Z}(t)$ é um processo auto-similar arbitrário, i.e.,

$$
\mathbf{Z}(t) \stackrel{d}{=} m^{-H} \mathbf{Z}(m t)
$$

onde $0<H<1$. O símbolo $\stackrel{d}{=}$ denota igualdade em distribuição. Supondo ainda que os incrementos de $\mathbf{Z}(t)$ são 
estacionários (i.e., $\mathbf{Z}(t+\tau)-\mathbf{Z}(t) \stackrel{d}{=} \mathbf{Z}(\tau)$, para $\forall t, \tau \geq 0$ ), o incremento do processo $\mathbf{A}(t)$ no intervalo $[t ; t+\tau]$ é dado por

$$
\begin{aligned}
\Delta \mathbf{A}(t ; t+\tau) & =\mathbf{A}(t+\tau)-\mathbf{A}(t) \\
& =\mu \tau+\gamma \mathbf{Z}(\tau) .
\end{aligned}
$$

O parâmetro $\tau$ representa a escala de tempo na qual o tráfego incremental é calculado. Cabe observar que o processo $\Delta \mathbf{A}(t ; t+\tau)$ também é estacionário - por simplicidade, ele será representado por $\Delta \mathbf{A}(\tau)$. Um processo envelope para $\Delta \mathbf{A}(\tau)$ pode ser definido como

$$
\Delta \widehat{A}(\tau)=\mu \tau+k \gamma \tau^{H}, \forall \tau \geq 0 .
$$

Este processo é denominado Processo de Chegada com Limitante Fracionário (Fractional Bounded Arrival Process - FBAP) [21]. Tal denominação advém do fato de o processo ser uma generalização do conhecido Processo de Chegada com Limitante Linear (Linear Bounded Arrival Process, LBAP), o qual é obtido para $H=0[25,26]$.

A probabilidade de violação do envelope $\Delta \widehat{A}(\tau)$ é dada por

$$
\begin{aligned}
\mathbb{P}\{\Delta \mathbf{A}(\tau)>\Delta \widehat{A}(\tau)\} & =\mathbb{P}\{\mathbf{Z}(1)>k\} \\
& =\bar{F}_{\mathbf{Z}}(k) .
\end{aligned}
$$

Este resultado é diferente daquele que seria obtido no caso do processo envelope do fBm proposto em [18]:

$$
\begin{aligned}
\mathbb{P}\{\Delta \mathbf{A}(\tau)>\widehat{A}(t+\tau)-\widehat{A}(t)\} & \\
& =\mathbb{P}\left\{\mathbf{Z}(\mathbf{1})>k \frac{(t+\tau)^{H}-t^{H}}{\tau^{H}}\right\} .
\end{aligned}
$$

Como, por suposição, os incrementos de tráfego são estacionários, o modelo FBAP representa melhor o comportamento do tráfego, dado que a probabilidade de violação é, neste caso, constante no tempo.

Os parâmetros do envelope FBAP podem ser obtidos da seguinte maneira: seja $\Delta A(t ; t+\tau)$ a realização do processo de incrementos $\Delta \mathbf{A}(t ; t+\tau)$. Os parâmetros $\mu \mathrm{e} \gamma$ podem ser obtidos diretamente da distribuição marginal de $\Delta \mathbf{A}(t ; t+\tau)$. Por exemplo, se a distribuição marginal de $\Delta \mathbf{A}(t ; t+\tau)$ é gaussiana, $\mu$ corresponde à média e $\gamma$ pode ser obtido do desvio padrão da série, dado por $\gamma \tau^{H}$. Para a obtenção do parâmetro de auto-similaridade $H$, existem diversos métodos [27, 28]. Finalmente, o parâmetro $k$ deve ser escolhido de modo a que se obtenha a probabilidade de violação desejada.

O uso do modelo FBAP é ilustrado na Figura 1. Neste exemplo, o tráfego corresponde a um traço de ruído gaussiano fracionário, com $10^{6}$ amostras, o qual foi gerado utilizando o método proposto em [29]. Os parâmetros do traço são $\mu=$ $0.8, \gamma=0.16$ e $H=0.8$. Os parâmetros $\mu, \gamma$ e $H$ do modelo FBAP são iguais aos parâmetros do traço, e o parâmetro $k$ é igual a 3.719 , o que resulta em uma probabilidade de violação menor do que $10^{-4}$.

Observe que o modelo FBAP representa bem o comportamento fractal do tráfego, mesmo em escalas de tempo eleva-

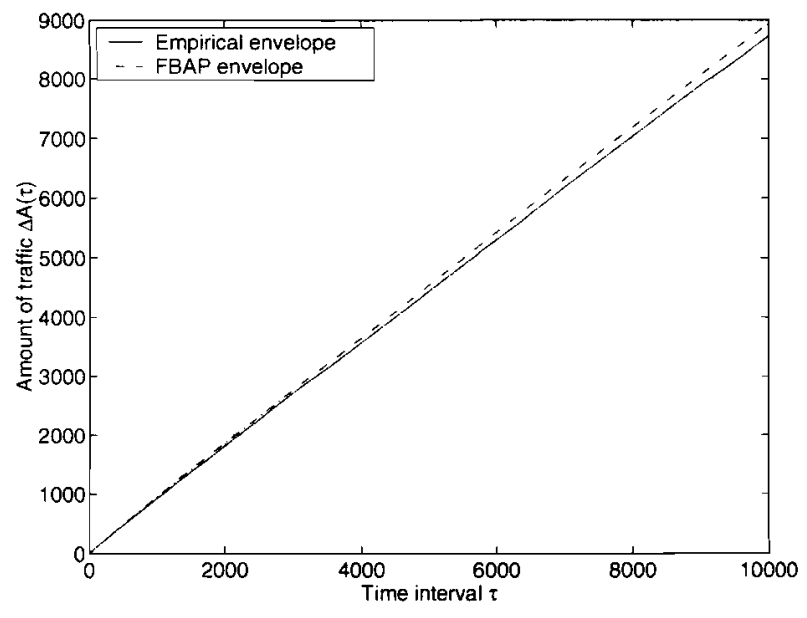

Figura 1. Validação do modelo FBAP. Os parâmetros do tráfego são $\mu=0.8, \gamma=0.16$ e $H=0.8$. Para o modelo FBAP, os parâmetros $\mu, \gamma$ e $H$ são iguais aos do tráfego, e o parâmetro $k$ é igual a 3.719 .

das. A diferença entre o envelope FBAP e o envelope empírico é devida a imprecisões na geração da série temporal e na estimação dos parâmetros fGn.

Para o caso de um traço de trafégo real, os parâmetros do modelo FBAP podem ser obtidos por meio do método do envelope empírico. Seja $\mathbf{A}[n]$ a série temporal que representa o traço para o qual se deseja obter o envelope, i.e.,

$$
\mathbf{A}[n]=\mathbf{A}(n T), n \in \mathbb{N}, T \geq 0 .
$$

Os incrementos discretos correspondentes a $\mathbf{A}[n]$ são dados por:

$$
\begin{aligned}
\Delta \mathbf{A}_{(i)}[n] & =\mathbf{A}[(n+1) i]-\mathbf{A}[n i] \\
& =\mu T i+\gamma \mathbf{Z}[i], n, i \in \mathbb{N},
\end{aligned}
$$

onde $\mathbf{Z}[i]$ é o processo discreto correspondente a $\mathbf{Z}(t)$. A realização do processo $\Delta \mathbf{A}_{(i)}[n]$ é dada por $\Delta A_{(i)}[n]$. Note que $\Delta A_{(i)}[n]$ corresponde justamente ao traço para o qual se deseja obter o envelope MFBAP.

O envelope empírico para o processo $\Delta \mathbf{A}_{(i)}[n]$ é definido como

$$
\Delta A^{(e)}[i]=\mu T i+Z^{(e)}[i],
$$

onde,

$$
\begin{gathered}
\mu=\frac{1}{T} \mathbb{E}\left\{\Delta A_{(1)}[n]\right\} . \\
Z^{(e)}[i]=\left\{x \in \mathbb{R}: \mathbb{P}\left\{\Delta \mathbf{A}_{(i)}[n]-\mu T i>x\right\}=\varepsilon\right\},
\end{gathered}
$$

onde $\epsilon$ é a probabilidade de violação desejada e, para cada escala de tempo, a probabilidade $\mathbb{P}\left\{\Delta \mathbf{A}_{(i)}[n]-\mu T i>x\right\}$ é obtida diretamente da série temporal.

O produto $k \gamma$ e o parâmetro $H$ podem ser obtidos por meio do ajuste de $\Delta \widehat{A}(i T)-\mu i T=k \gamma(i T)^{H}$ de modo que esta função limite por cima $Z^{(e)}[i]$ até uma escala de tempo suficientemente grande. Contudo, esta abordagem geralmente leva a um envelope FBAP muito conservador. Uma abordagem mais realista se baseia na escolha de valores de $k \gamma$ e de 
$H$ para os quais uma pequena violação do envelope empírico (12) é tolerada. Por exemplo, isto pode ser obtido resolvendo

$$
\begin{gathered}
\{k \gamma, H\}=\arg \quad \min _{0 \leq H \leq 1} \\
k \gamma \geq 0 \\
\left.\left\{\frac{1}{2} \sum_{i=1}^{N}\left[f\left(\log Z^{(e)} \mid i\right]-\log k \gamma-H \log i\right)\right]^{2}\right\},
\end{gathered}
$$

onde

$$
f(x)= \begin{cases}x, & x<0 \\ w x, & x \geq 0, w>1 .\end{cases}
$$

O valor de $w$ deve ser escolhido de modo a se obter uma solução de compromisso entre a violação de (12) e o conservadorismo do envelope. Em alguns casos, obtém-se melhor resultado associando (15) com algum outro procedimento de estimação. Por exemplo, o produto $k \gamma$ pode ser dado por

$$
k \gamma=\max _{n} \Delta A[n]
$$

e o parâmetro $H$ estimado por meio de

$$
\begin{aligned}
& H=\arg \min _{0 \leq H \leq 1} \\
& \left\{\frac{1}{2} \sum_{i=1}^{N}\left[f\left(\log Z^{(e)}[i]-\log k \gamma-H \log i\right)\right]^{2}\right\} .
\end{aligned}
$$

Diversos traços de tráfego real foram utilizados para validação do modelo FBAP, conforme indicado na Tabela 1. Os traços BCPAUG89, BCPOCT89, ВСОCT89EXT, BCOCT 89EXT4, LBLTCP3, LBLPKT4-5 e DECPKT2-4 correspondem a traços de tráfego de redes reais, que apresentam características fractais e que são normalmente utilizados no estudo de modelagem de tráfego [30, 1, 2, 4, 31].

Os traços originais foram pré-processados para criar sequiências com até $1 \times 10^{6}$ amostras, que representam a quantidade de tráfego (em bytes) que flui através da rede durante um dado intervalo de tempo. Assumiram-se intervalo de $10 \mathrm{~ms}$ para o caso dos traços BCPAug89, BCPOCT89, BCOCT89Ext e BCOCT89ExT4. Para os traços LBLTCP3, LBLPKT4-5 e DECPKT2-4, assumiram-se intervalos de $100 \mathrm{~ms}$.

Os demais traços correspondem a seqüências de vídeo MPEG, que também apresentam comportamento fractal [5, 8]. Cada traço consiste em uma seqüência discreta de $4 \times 10^{4}$ amostras, sendo que cada amostra corresponde ao tamanho (em bytes) de um quadro de vídeo MPEG completo. Dado que os quadros são gerados a uma taxa de $20 \mathrm{fps}$, cada amostra corresponde à quantidade de tráfego (em bytes) que flui através da rede durante um intervalo de $50 \mathrm{~ms}$.

Os parâmetros do modelo FBAP foram escolhidos de modo a se obter uma probabilidade de violação de $10^{-4}$. O parâmetro $\mu$ foi obtido utilizando (13). O produto $k \gamma$ e o parâmetro $H$ foram obtidos utilizando (15) ou por meio de (16) e (17). Nos resultados, estas abordagens são denominadas por Método 1 e Método 2, respectivamente. Em ambos os casos, foi assumido $w=10$.

Na Tabela 1, são indicados os parâmetros obtidos para cada traço, bem como o método utilizado para obter $k \gamma$ e $H$. As
Figs. 2 a 4 apresentam a relação $\log Z^{(e)}[i] \times \log i$ correspondente aos traços BOND, FUSS1 e DECPKT4. Os envelopes empíricos e os envelopes FBAP para estes traços são mostrados nas Figs. 5 a 7 , sendo o erro relativo entre o envelope empírico e o modelo FBAP dado por

$$
e[i]=\frac{\Delta \widehat{A}(i T)-\Delta A^{(e)}[i]}{\Delta A^{(e)}[i]} .
$$

Os resultados obtidos para os demais traços podem ser encontrados na referência [32]. Observe que o modelo FBAP representa bem o comportamento fractal do tráfego, em particular quando ele é consistente com o conceito de autosimilaridade estatística.

\begin{tabular}{|c||c|c|c|c|}
\hline Trace & Method & $\mu$ & $k \gamma$ & $H$ \\
\hline \hline BCOCT89EXT & 2 & $7.49 \times 10^{2}$ & $5.12 \times 10^{4}$ & 0.676 \\
\hline BCOCT89EXT4 & 2 & $6.22 \times 10^{3}$ & $1.90 \times 10^{5}$ & 0.949 \\
\hline BCPAUG89 & 1 & $1.38 \times 10^{5}$ & $4.54 \times 10^{5}$ & 0.759 \\
\hline BCPOCT89 & 2 & $3.63 \times 10^{5}$ & $6.24 \times 10^{5}$ & 0.911 \\
\hline ASTERIX & 1 & $5.59 \times 10^{5}$ & $1.56 \times 10^{6}$ & 0.740 \\
\hline ATP & 2 & $5.47 \times 10^{5}$ & $1.54 \times 10^{6}$ & 0.699 \\
\hline BOND & 2 & $6.08 \times 10^{5}$ & $1.41 \times 10^{6}$ & 0.659 \\
\hline DECPKT2 & 2 & $2.38 \times 10^{5}$ & $3.79 \times 10^{5}$ & 0.793 \\
\hline DECPKT3 & 2 & $1.81 \times 10^{5}$ & $3.04 \times 10^{5}$ & 0.766 \\
\hline DECPKT4 & 2 & $2.63 \times 10^{5}$ & $3.09 \times 10^{5}$ & 0.789 \\
\hline DINO & 2 & $3.27 \times 10^{5}$ & $9.66 \times 10^{5}$ & 0.723 \\
\hline FUSS1 & 1 & $6.78 \times 10^{5}$ & $1.55 \times 10^{6}$ & 0.639 \\
\hline FUSS2 & 2 & $6.28 \times 10^{5}$ & $1.92 \times 10^{6}$ & 0.774 \\
\hline I.AMBS & 2 & $1.83 \times 10^{5}$ & $1.02 \times 10^{6}$ & 0.710 \\
\hline LBLPKT4 & 1 & $3.64 \times 10^{4}$ & $2.57 \times 10^{5}$ & 0.736 \\
\hline LBLPKT5 & 2 & $2.61 \times 10^{4}$ & $2.30 \times 10^{5}$ & 0.718 \\
\hline LBLTCP3 & 2 & $3.39 \times 10^{4}$ & $3.76 \times 10^{5}$ & 0.814 \\
\hline MOVIF & 2 & $3.57 \times 10^{5}$ & $1.04 \times 10^{6}$ & 0.641 \\
\hline MRBEAN & 2 & $4.41 \times 10^{5}$ & $1.59 \times 10^{6}$ & 0.777 \\
\hline MTV1 & 2 & $6.15 \times 10^{5}$ & $2.20 \times 10^{6}$ & 0.797 \\
\hline MTV2 & 2 & $4.95 \times 10^{5}$ & $2.61 \times 10^{6}$ & 0.769 \\
\hline NEWS1 & 2 & $5.17 \times 10^{5}$ & $1.47 \times 10^{6}$ & 0.694 \\
\hline NEWS2 & 1 & $3.84 \times 10^{5}$ & $2.00 \times 10^{6}$ & 0.733 \\
\hline RACF & 1 & $7.69 \times 10^{5}$ & $2.03 \times 10^{6}$ & 0.732 \\
\hline SBOWL & 2 & $5.88 \times 10^{5}$ & $1.20 \times 10^{6}$ & 0.727 \\
\hline SIMPSONS & 2 & $4.64 \times 10^{5}$ & $1.19 \times 10^{6}$ & 0.702 \\
\hline STAR2 & 2 & $2.33 \times 10^{5}$ & $1.16 \times 10^{6}$ & 0.729 \\
\hline TALK1 & 2 & $3.63 \times 10^{5}$ & $8.96 \times 10^{5}$ & 0.717 \\
\hline TALK2 & 2 & $4.48 \times 10^{5}$ & $1.22 \times 10^{6}$ & 0.766 \\
\hline TERMINATOR & 1 & $2.73 \times 10^{5}$ & $5.11 \times 10^{5}$ & 0.626 \\
\hline & & & & \\
\hline
\end{tabular}

Tabela 1. Parâmetros do envelope FBAP correspondente aos traços analisados.

\section{ANÁLISE DE FILAS PARA TRÁFEGO FBAP}

Seja um sistema de filas, no qual os incrementos de tráfego na entrada são representados por $\Delta \mathbf{A}(t ; t+\tau)$, o backlog é representado por $\mathbf{Q}(t)$, e o servidor tem taxa constante, dada por $g$. De modo a garantir a estabilidade do sistema, supõe-se que a taxa do servidor é maior do que a taxa média do tráfego de entrada, dada por $\mu$. 


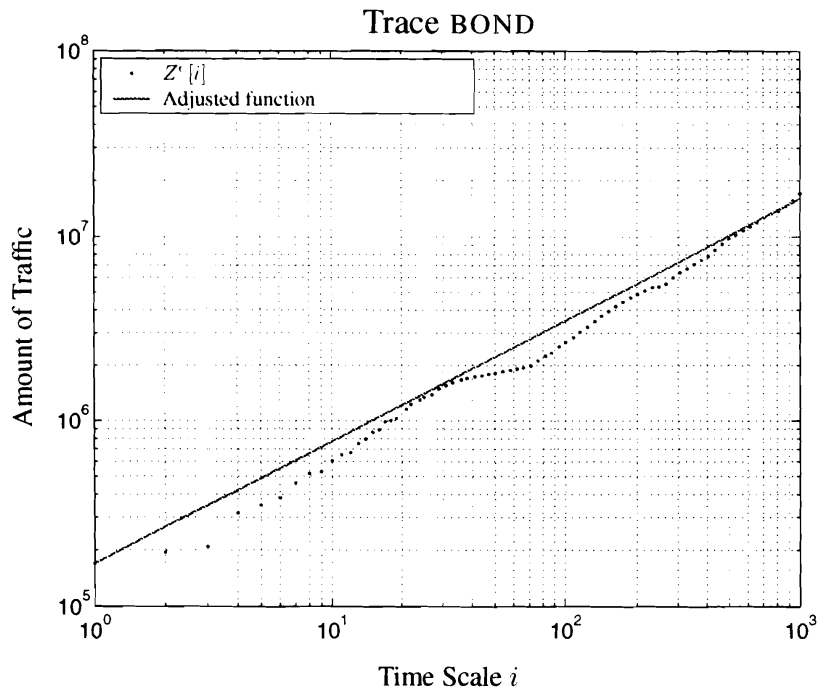

Figura 2. Relação $\log Z^{(e)}[i] \times \log i$ para o traço BOND, e sua aproximação pelo modelo FBAP.

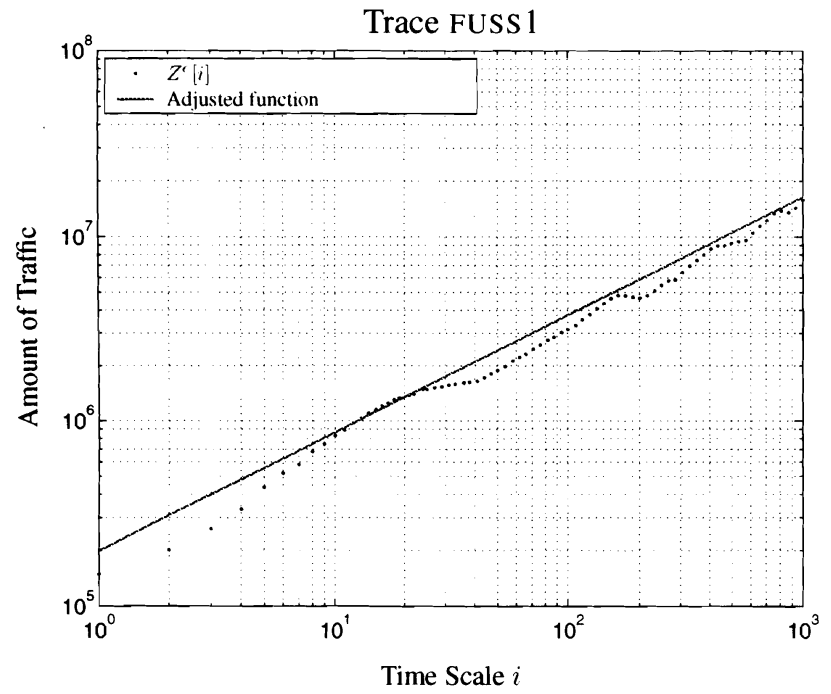

Figura 3. Relação $\log Z^{(e)}[i] \times \log i$ para o traço FUSS 1, e sua aproximação pelo modelo FBAP.

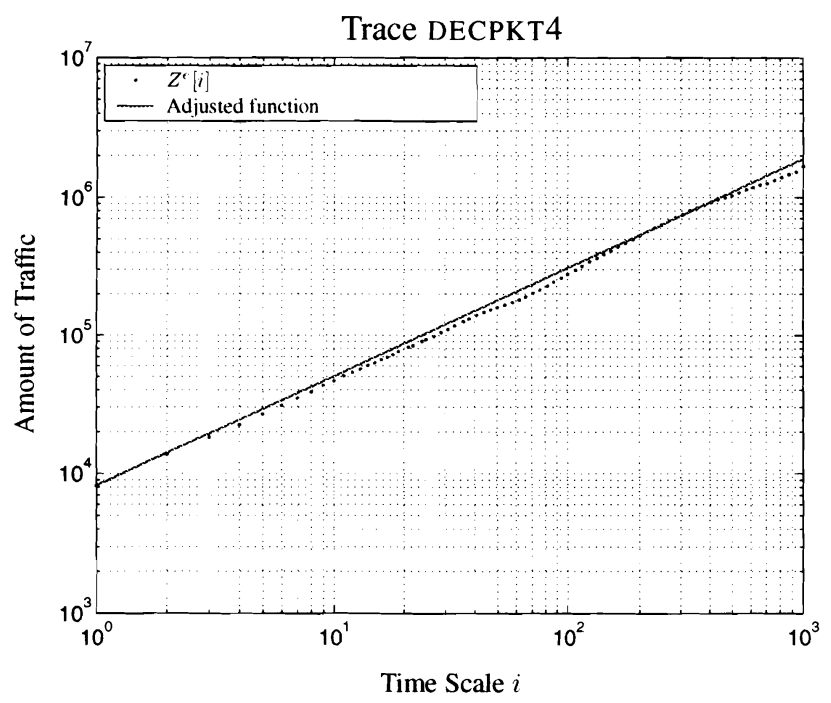

Figura 4. Relação $\log Z^{(e)}[i] \times \log i$ para o traço LBLTCP3, e sua aproximação pelo modelo FBAP.
Suponha que o $i$-ésimo ciclo ativo do servidor inicie no instante $t_{i}$. Entenda-se por ciclo ativo o período no qual o tráfego de entrada é continuamente acumulado na fila. Assim,

$$
\mathbf{Q}\left(t_{i}+\tau\right)=\Delta \mathbf{A}\left(t_{i} ; t_{i}+\tau\right)-g \tau, 0 \leq \tau<\tau_{i}^{b u s y},
$$

onde $\tau_{i}^{b u s y}$ é a duração do $i$-ésimo ciclo ativo do servidor.

Supondo que o tráfego de entrada é FBAP, um limitante superior para $\mathbf{Q}\left(t_{i}+\tau\right)$ pode ser definido como sendo

$$
\begin{aligned}
\widehat{Q}(\tau) & =\Delta \widehat{A}(\tau)-g \tau \\
& =(\mu-g) \tau+k \gamma \tau^{H}, 0 \leq \tau<\tau^{b u s y} .
\end{aligned}
$$

onde $\tau^{\text {busy }}$ é dado por $\arg _{\tau>0}\{\widehat{Q}(\tau)=0\}$. A probabilidade de que $\mathbf{Q}\left(t_{i}+\tau\right)$ exceda $\widehat{Q}(\tau)$ é dada por

$$
\begin{aligned}
\mathbb{P}\left\{\mathbf{Q}\left(t_{i}+\tau\right)>\widehat{Q}(\tau)\right\} & =\mathbb{P}\{\Delta \mathbf{A}(\tau)>\Delta \widehat{A}(\tau)\} \\
& =\bar{F}_{\mathbf{z}}(k),
\end{aligned}
$$

Para $0<H<1$, o envelope $\widehat{Q}(\tau)$ é $\cap$-convexo em $\tau \geq 0$. Um limitante superior para o máximo backlog do sistema de filas pode, então, ser definido como

$$
\begin{aligned}
Q^{*} & =\max _{0 \leq \tau \leq \tau^{b u s y}} \widehat{Q}(\tau) \\
& =(g-\mu)^{\frac{H}{H-1}}(k \gamma)^{\frac{1}{1-H}} H^{\frac{H}{1-H}}(1-H) .
\end{aligned}
$$

A escala de tempo para a qual se obtém $Q^{*}$ é denominada escala de tempo máxima $[18,33]$. A probabilidade de violação do limitante $Q^{*}$ no instante $t_{i}+\tau$ é dada por

$$
\begin{aligned}
\mathbb{P}\left\{\mathbf{Q}\left(t_{i}+\tau\right)>Q^{*}\right\} & \leq \mathbb{P}\left\{\mathbf{Q}\left(t_{i}+\tau\right)>\widehat{Q}(\tau)\right\} \\
& =\bar{F}_{\mathbf{Z}}(k) .
\end{aligned}
$$

A probabilidade de violação do limitante $Q^{*}$ em qualquer instante de tempo durante o ciclo ativo é, então, dada por [21]

$$
\begin{aligned}
\mathcal{P} & \geq \sup _{\tau \geq 0} \mathbb{P}\left\{\mathbf{Q}\left(t_{i}+\tau\right)>Q^{*}\right\} \\
& =\bar{F}_{\mathbf{Z}}(k) .
\end{aligned}
$$

No caso do tráfego Browniano fracionário, a relação acima converge para uma igualdade se $Q^{*}$ é suficientemente grande, o que equivale a uma probabilidade de violação suficientemente pequena [34]. Dado o limitante (20), o máximo retardo introduzido no tráfego pelo sistema de filas pode ser limitado por $D^{*}=Q^{*} / g$. Além disso, a relação (20) pode ser utilizada para calcular a taxa de servidor para a qual o backlog é limitado a $Q^{*}$ com probabilidade igual a $1-\mathcal{P}$ :

$$
g=\mu+(k \gamma)^{\frac{1}{H}} H(1-H)^{\frac{1-H}{H}}\left(Q^{*}\right)^{\frac{H-1}{H}} .
$$

Nesta relação, o valor de $g$ pode ser considerado como sendo a banda efetiva do tráfego FBAP. Observa-se que (23) também representa a banda efetiva do processo envelope do fBm [19]. 

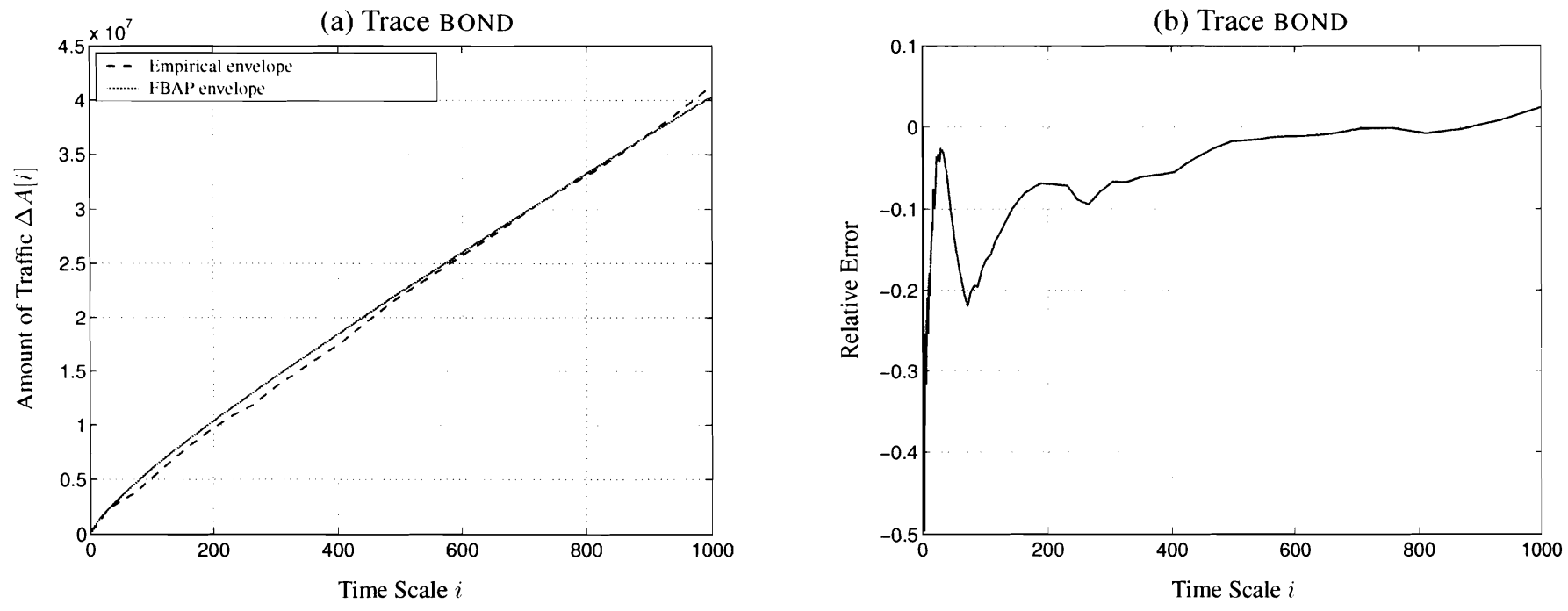

Figura 5. Representação do traço BOND utilizando o envelope FBAP: (a) envelope empírico e FBAP; (b) erro relativo decorrente do uso do envelope FBAP.
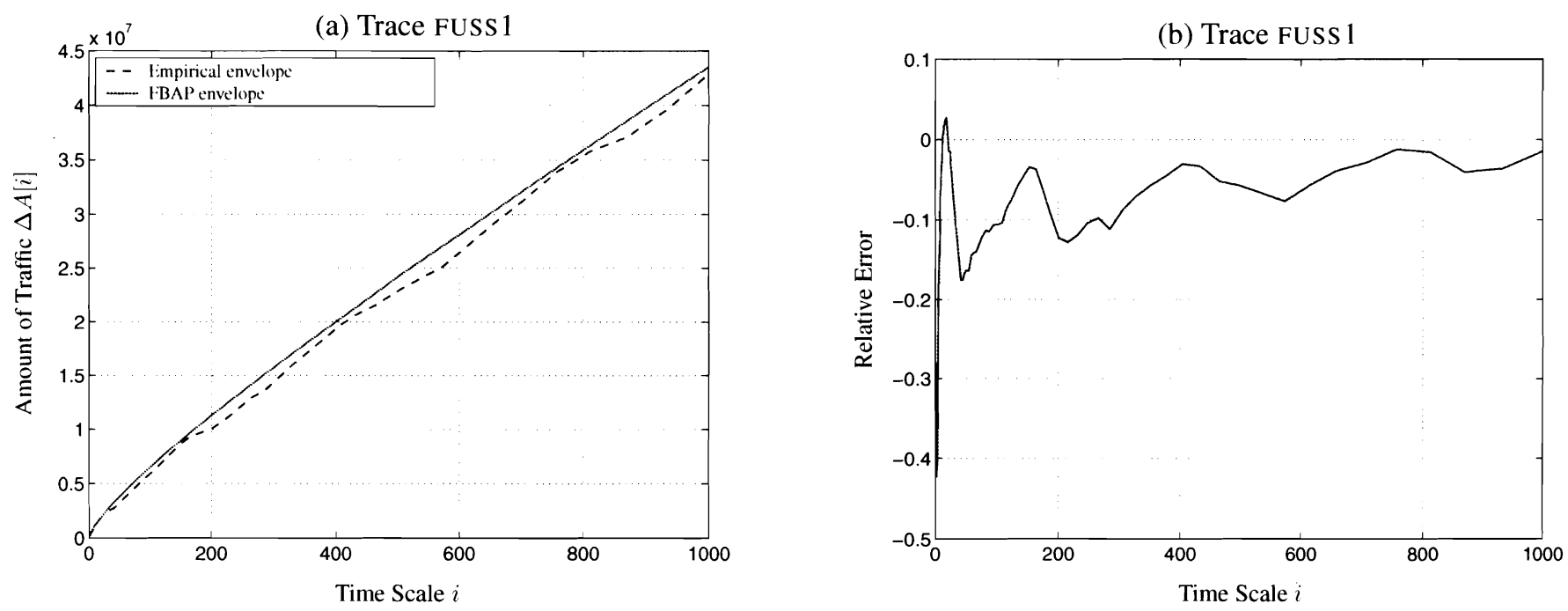

Figura 6. Representação do traço FUSS 1 utilizando o envelope FBAP: (a) envelope empírico e FBAP; (b) erro relativo decorrente do uso do envelope FBAP.
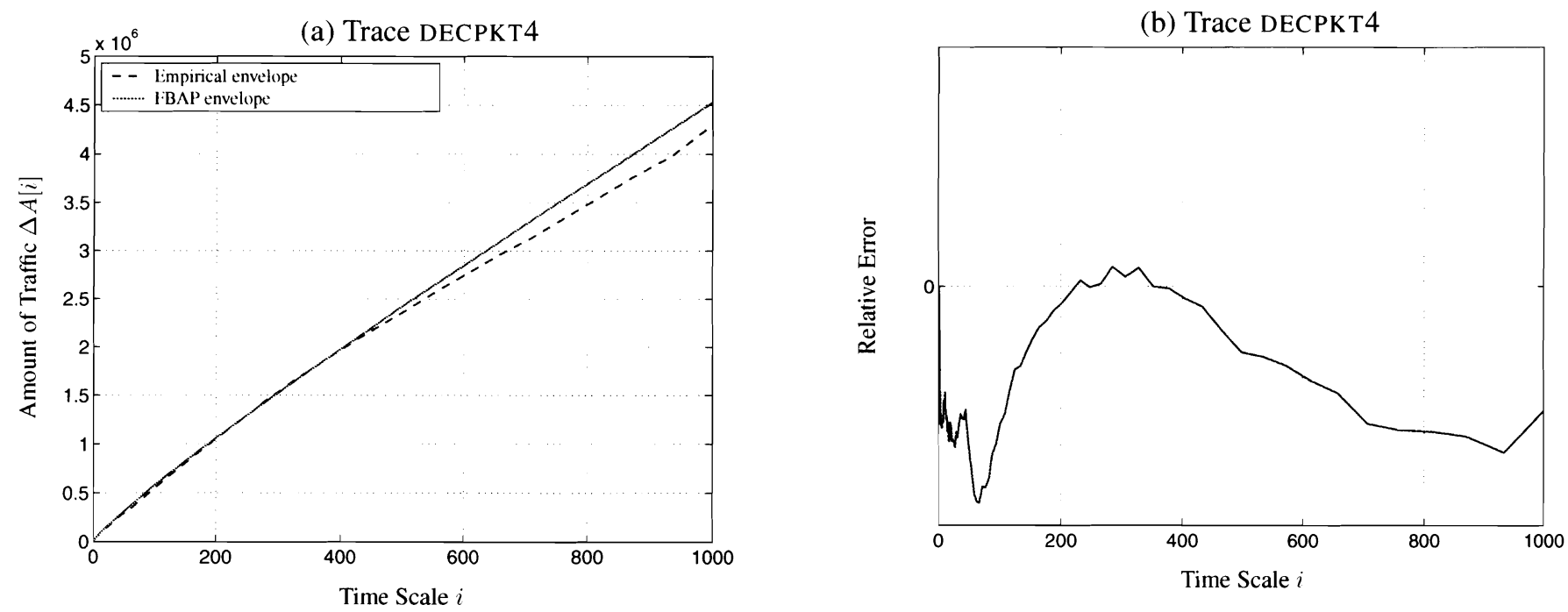

Figura 7. Representação do traço DECPKT4 utilizando o envelope FBAP: (a) envelope empírico e FBAP; (b) erro relativo decorrente do uso do envelope FBAP. 


\section{POLICIAMENTO DE TRÁFEGO FBAP UTILIZANDO O ALGORITMO DO BALDE FURADO}

A partir dos resultados obtidos na seção anterior, pode-se concluir que o uso de um mecanismo de policiamento que restrinja o tráfego a um envelope FBAP permite estabelecer garantias de desempenho em um sistema de filas. Contudo, a obtenção de tal mecanismo não é trivial, pois a nãolinearidade do modelo FBAP implica em

$$
\Delta \widehat{A}(m \tau) \leq m \Delta \widehat{A}(\tau) .
$$

A condição de igualdade é obtida apenas se $m=1$ ou se $H=0$. Tal relação indica que, em geral, um mecanismo de policiamento de janelas não pode ser empregado para policiar tráfego FBAP, pois não é suficiente policiar $m$ intervalos consecutivos de tamanho $\tau$ para garantir que todo $o$ intervalo de tamanho $m \tau$ seja policiado.

Nesta seção, estuda-se o policiamento de tráfego FBAP utilizando o algoritmo do Balde Furado. Este algoritmo restringe o tráfego incremental a um envelope LBAP, dado por

$$
\Delta \widehat{A}_{\mathrm{LBAP}}(\tau)=r \tau+s
$$

onde $r$ é a taxa de drenagem e $s$ é o tamanho do balde. $O$ algoritmo do Balde Furado pode ser representado por um sistema de filas, no qual o balde é representado por uma fila de comprimento máximo $s$, continuamente servida a uma taxa igual a $r$. Quando uma quantidade qualquer de tráfego chega ao mecanismo de policiamento, a mesma quantidade de permissões (tokens) é inserida na fila. Se a fila não transborda, o tráfego está em conformidade e é imediatamente transferido à saída do mecanismo de policiamento. Caso contrário, descarta-se a quantidade de tráfego correspondente ao transbordo.

Seja $\Delta \hat{\widehat{A}}_{s}(\tau)=\mu_{s} \tau+k_{s} \gamma_{s} \tau^{H_{s}}$ o envelope FBAP especificado para o tráfego a ser policiado. Da análise de filas desenvolvida na seção anterior, depreende-se que uma dada probabilidade de descarte de tráfego pode ser obtida fazendose a taxa de drenagem igual à banda efetiva do tráfego, e o tamanho do balde igual ao correspondente limitante de backlog. De (20),

$$
s=\left(r-\mu_{s}\right)^{\frac{H_{s}}{H_{s}-1}}\left(k_{s} \gamma_{s}\right)^{\frac{1}{1-H_{s}}} H_{s}^{\frac{H_{s}}{1-H_{s}}}\left(1-H_{s}\right)
$$

Em [19], uma relação similar foi obtida por meio de outra abordagem. Seja $\Delta \mathbf{A}_{i}(\tau)=\mu_{i} \tau+\gamma_{i} \mathbf{Z}_{i}(\tau)$ o tráfego a ser policiado, e $\mathcal{P}_{i}$ a probabilidade de descarte. Quando $\mu_{i} \geq \mu_{s}$, esta probabilidade é igual a 1. Por outro lado, quando $\mu_{s} \geq$ $\mu_{i}$, a probabilidade $\mathcal{P}_{i}$ é limitada por

$$
\begin{aligned}
\mathcal{P}_{i} & =\mathbb{P}\left\{\sup _{\tau \geq 0} \mathbf{Q}_{i}(t+\tau)>s\right\} \\
& \geq \sup _{\tau \geq 0} \mathbb{P}\left\{\mathbf{Z}_{i}(1)>\frac{s+\left(r-\mu_{i}\right) \tau}{\gamma_{i} \tau^{H_{i}}}\right\} \\
& =\bar{F}_{\mathbf{Z}_{i}}\left[\frac{\left(r-\mu_{i}\right)^{H_{i}}}{\gamma_{i} H_{i}^{H_{i}}\left(1-H_{i}\right)^{1-H_{i}}} s^{1-H_{i}}\right] .
\end{aligned}
$$

Quando o tráfego de entrada satisfaz as especificações, a probabilidade de descarte é dada por

$$
\mathcal{P}_{i} \geq \bar{F}_{\mathbf{z}_{i}}\left(k_{s}\right)
$$

No caso de a distribuição marginal do tráfego ser gaussiana, a relação acima converge para uma igualdade quando a probabilidade de violação é suficientemente pequena , i.e.,

$$
\mathcal{P}_{i} \simeq \bar{F}_{\mathbf{Z}_{i}}\left(k_{s}\right)
$$

De modo a garantir uma dada probabilidade de descarte de tráfego em conformidade, os parâmetros do algoritmo do Balde Furado devem ser escolhidos satisfazendo (25) e (27).

Finalmente, cabe observar que o tráfego que deixa o mecanismo de policiamento não é FBAP. Para sua representação, deve-se considerar o envelope LBAP correspondente, visto que o algoritmo do Balde Furado admite alguma violação de $\mu_{s}, \gamma_{s}$ e $H_{s}$, desde que esta não implique na violação do envelope LBAP subjacente.

O uso do algoritmo do Balde Furado para o policiamento de tráfego FBAP é ilustrado na Fig. 8 a 9. O tráfego de entrada é dado pelo traço DECPKT2-TCP [4]. Este traço foi pré-processado de modo a criar a sequiência utilizada nas simulações, na qual cada amostra corresponde à quantidade de tráfego (em bytes) que chega ao mecanismo de policiamento durante um intervalo de $10 \mathrm{~ms}$. Em cada intervalo, supõe-se que a distribuição temporal do tráfego é uniforme, o que reduz a incidência de surtos e dificulta a detecção de violações pelo algoritmo do Balde Furado.

O envelope FBAP correspondente ao traço é mostrado na Fig. 8. Os parâmetros do modelo são $\mu_{s}=2.38 \times 10^{5}$, $k_{s} \gamma_{s}=3.85 \times 10^{5}$ e $H_{s}=0.796$, o que resulta em uma probabilidade de violação de $10^{-4}$. Assumindo um sistema de filas simples, a relação entre o limitante para o máximo backlog e a taxa de serviço é mostrada na Fig. 9(a). Note que o máximo backlog cai rapidamente à medida em que a taxa de serviço aumenta, o que indica que o backlog pode ser pequeno mesmo para o caso de o tráfego ser servido a uma taxa próxima da taxa média.

Os valores máximos para o backlog quando o tráfego é policiado pelo algoritmo do Balde Furado e pelo algoritmo FLB também são mostrados na Fig. 9(a). As taxas de descarte correspondentes ao algoritmo FLB e ao algoritmo do Balde Furado são mostradas na Fig. 9(b). No caso do algoritmo do Balde Furado, a taxa de drenagem $r$ é igual à taxa de serviço, e o tamanho do balde $s$ é dado por (25). No caso do algoritmo FLB, os parâmetros são os mesmos do modelo FBAP.

A partir da Fig. 9(a), pode-se observar que o limitante de backlog nunca é violado quando o tráfego é policiado pelo algoritmo do Balde Furado. Por outro lado, pode haver violação quando o algoritmo FLB é utilizado. Este resultado confirma a análise do FLB realizada em [21], e mostra que este algoritmo não é capaz de suportar o oferecimento de garantias de desempenho, o que é possível com o algoritmo do Balde Furado. Além disso, o algoritmo do Balde Furado sempre descarta menos tráfego, o que resulta em menores perdas de tráfego para o usuário. 


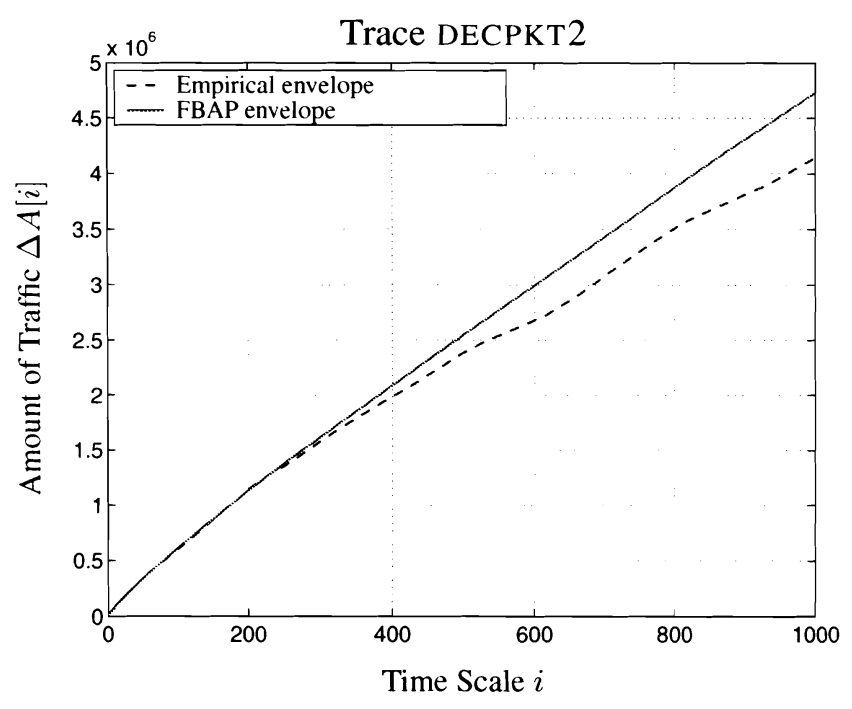

Figura 8. Envelope FBAP para o traço DECPKT2-TCP.

(a) Trace DECPKT2

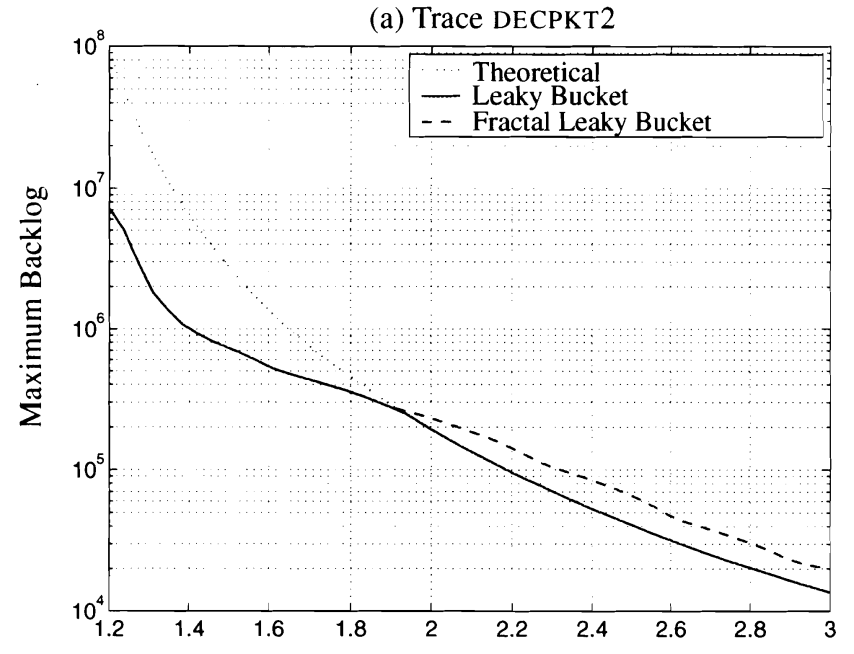

(b) Trace DECPKT2

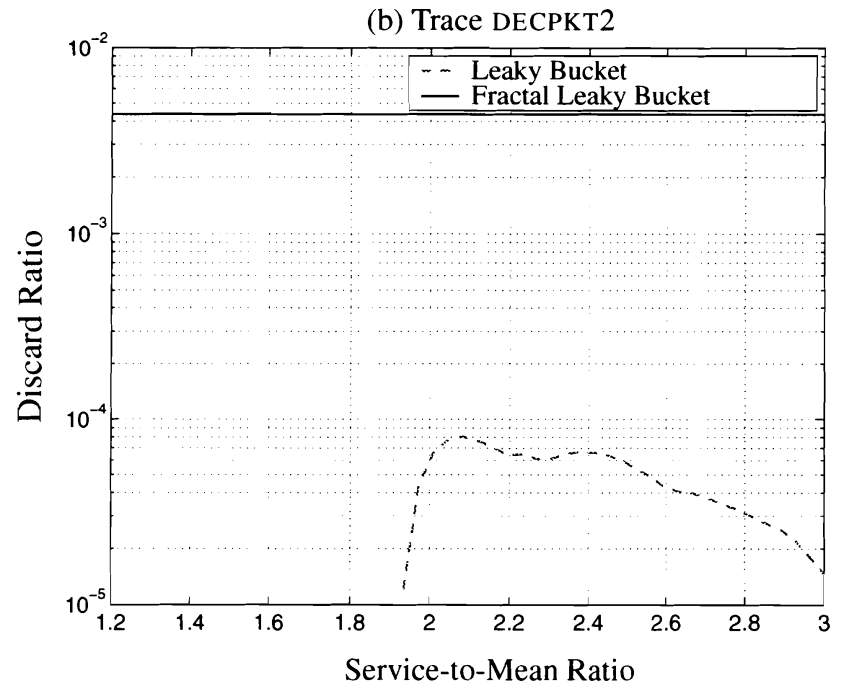

Figura 9. Resultados de simulação do policiamento de tráfego FBAP utilizando o algoritmo do Balde Furado e o algoritmo do Balde Furado Fractal. (a) Máximo backlog; (b) Taxa de descarte.

\section{O PROCESSO DE CHEGADA COM LIMITANTE MULTIFRACTAL}

Nesta seção, propõe-se um novo processo envelope para a modelagem de tráfego fractal. Seja o tráfego acumulado até $\mathrm{o}$ instante $t$ dado por

$$
\mathbf{A}(t)=\mu t+\gamma \mathbf{Z}(t), t \geq 0, \mathbf{Z}(0)=0,
$$

onde $\mathbf{Z}(t)$ é um processo estocástico multifractal com incrementos estacionários. Em particular, assume-se que um processo $\mathbf{Z}(t)$ com incrementos estacionários é um processo multifractal se a seguinte relação é satisfeita

$$
\mathbf{Z}(m t) \stackrel{d}{=} \mathbf{C}(m) \mathbf{Z}(t), m, t \geq 0,
$$

onde $\mathbf{Z}(t)$ e $\mathbf{C}(m)$ são dois processos estocásticos independentes. A partir de (29), pode-se concluir que

$$
\mathbf{Z}(t) \stackrel{d}{=} \mathbf{C}(t) \mathbf{Z}(1), t \geq 0 .
$$

Note que $\mathbf{C}(0)=0$, pois $\mathbf{Z}(0)=0$. Além disso, dado que os incrementos de $\mathbf{Z}(t)$ são estacionários,

$$
\begin{aligned}
\mathbf{Z}(t+\tau)-\mathbf{Z}(t) & \stackrel{d}{=} \mathbf{Z}(\tau)-\mathbf{Z}(0) \\
& \stackrel{d}{=} \mathbf{Z}(1)[\mathbf{C}(\tau)-\mathbf{C}(0)] \\
& \stackrel{d}{=} \mathbf{Z}(1) \mathbf{C}(\tau), \tau, t \geq 0
\end{aligned}
$$

Os incrementos do processo $\mathbf{A}(t)$ no intervalo $[t ; t+\tau]$ são, portanto, dados por

$$
\begin{aligned}
\Delta \mathbf{A}(t ; t+\tau) & =\mathbf{A}(t+\tau)-\mathbf{A}(t) \\
& =\mu \tau+\gamma \mathbf{Z}(1) \mathbf{C}(\tau) .
\end{aligned}
$$

Note que o processo $\Delta \mathbf{A}(\cdot)$ também é estacionário. Um processo envelope para este processo pode ser definido como

$$
\Delta \widehat{A}(\tau)=\mu \tau+k \gamma \widehat{C}(\tau), \forall \tau \geq 0,
$$

onde $\widehat{C}(\tau)$ é um envelope para o processo $\mathbf{C}(\tau)$. O processo envelope $\Delta \widehat{A}(\tau)$ será denominado Processo de Chegada com Limitante Multifractal (Multifractal Bounded Arrival Process - MFBAP). Note que este processo pode ser visto como uma generalização do Processo de Chegada com Limitante Fracionário, o qual é obtido quando $\widehat{C}(\tau)=\tau^{H}, 0<H<1$.

Para o cálculo da probabilidade de violação do envelope $\Delta \widehat{A}(\tau)$, supor-se-á que o envelope $\widehat{C}(\tau)$ é determinístico. Embora seja aparentemente restritiva do ponto de vista matemático, tal suposição é razoável pois, na prática, capacidade de geração e de transporte de tráfego é sempre limitada. Isto significa que, para $\tau$ finito, a distribuição marginal de $\mathbf{C}(\tau)$ tem suporte finito. Assim, um envelope determinístico para este processo sempre pode ser obtido.

Assim, a probabilidade de que os incrementos de tráfego excedam o envelope $\Delta \widehat{A}(\tau)$ é dada por

$$
\begin{aligned}
\mathbb{P}\{\Delta \mathbf{A}(\cdot)>\Delta \widehat{A}(\tau)\} \\
\quad=\mathbb{P}\{\mu \tau+\gamma \mathbf{Z}(1) \mathbf{C}(\tau)>\mu \tau+k \gamma \widehat{C}(\tau)\} \\
\leq \mathbb{P}\{\mathbf{Z}(1)>k\} \\
=\bar{F}_{\mathbf{Z}}(k),
\end{aligned}
$$


onde $\bar{F} \mathbf{Z}(\cdot)$ representa a função distribuição residual da variável aleatória $\mathbf{Z}(1)$.

O envelope MFBAP para um dado traço de tráfego pode ser obtido a partir da definição de envelope empírico apresentada na Seção 2. O parâmetro $\mu$ é dado por (13). O envelope $\widehat{C}(\tau)$ é obtido através da análise gráfica da relação $\log Z^{(e)}[i] \times \log i$, escolhendo-se uma função que seja capaz de reproduzir o comportamento de $Z^{(e)}[i]$.

Para a validação do modelo MFBAP, foram utilizados os mesmos traços de tráfego real da Seção 2. Para os envelopes, escolheu-se uma probabilidade de violação $\varepsilon$ igual a $10^{-4}$. As Figs. 10 a 12 apresentam a relação $\log Z^{(e)}[i] \times \log i$ correspondente aos traços ATP, TALK1 e LBLTCP3. Note que, nos três casos, esta relação é aproximadamente logsigmoidal. Por isso, ela não pode ser adequadamente representada por meio de modelos auto-similares, para os quais esta relação é linear. Por exemplo, no caso do modelo FBAP, o termo que aproxima $Z^{(e)}[i]$ é dado por $k \gamma \tau^{H}$, que, no plano $\log -\log$, resulta na relação linear

$$
\log k \gamma \tau^{H}=\log k \gamma+H \log \tau
$$

As Figs. 10 a 12 mostram a aproximação obtida no caso do modelo FBAP. É possível concluir que, para garantir a probabilidade de violação especificada para o envelope, o modelo FBAP tende a ser conservador. Por outro lado, a relação $\log Z^{(e)}[i] \times \log i$ de cada um dos traços pode ser representada utilizando um modelo MFBAP, no qual

$$
\widehat{C}(\tau)=\tau^{H(\tau)}
$$

onde,

$$
H(\tau)=H_{0}+\gamma_{H} \exp \left\{-\frac{\left[\ln (\tau)-\mu_{H}\right]^{2}}{2 \sigma_{H}^{2}}\right\}
$$

É importante observar que o produto $k \gamma$ pode ser interpretado como um único parâmetro do modelo MFBAP proposto, dado por (32), (34) e (35). Assim, este modelo requer apenas 6 parâmetros para a caracterização do tráfego, o que assegura sua parcimônia.

As Figs. 10 a 12 mostram, para cada um dos traços já citados, a aproximação obtida no caso do modelo MFBAP. Os parâmetros do modelo para estes e para os demais traços analisados se encontram na Tabela 2. Os resultados obtidos para os demais traços podem ser encontrados na referência [32]. Neste caso, os resultados obtidos são significativamente melhores do que aqueles obtidos no caso do modelo FBAP. Isto se torna mais claro observando as Figs. 13 a 15, nas quais são apresentados o envelope empírico, o envelope FBAP, o envelope MFBAP e o erro relativo correspondente a cada um dos modelos são apresentados.

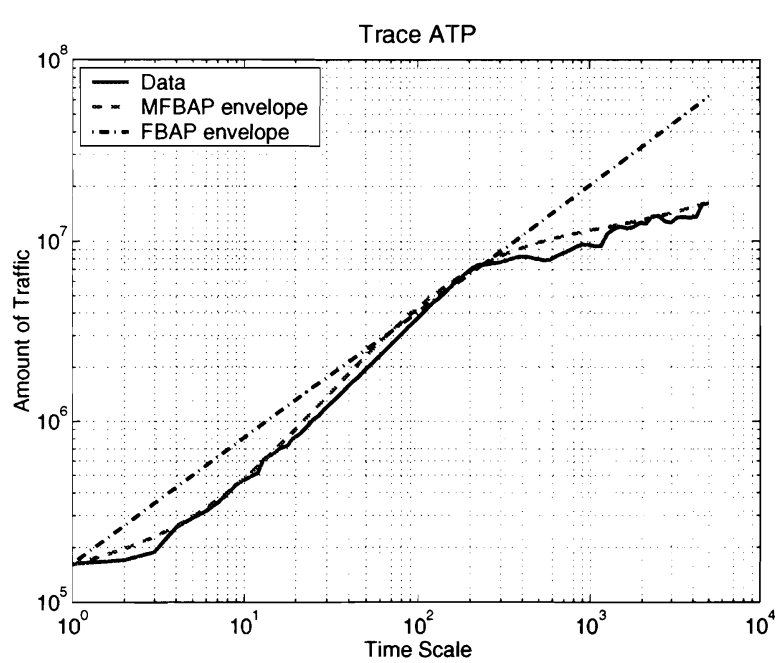

Figura 10. Relação $\log Z^{(e)}[i] \times \log i$ para o traço ATP, e sua aproximação para o caso dos modelos FBAP e MFBAP.

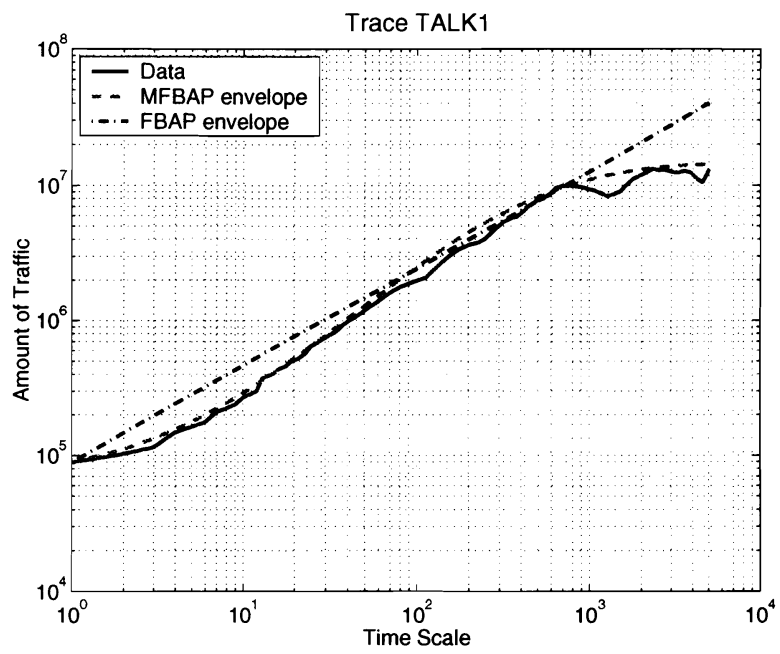

Figura 11. Relação $\log Z^{(e)}[i] \times \log i$ para o traço TALK 1 , e sua aproximação para o caso dos modelos FBAP e MFBAP.

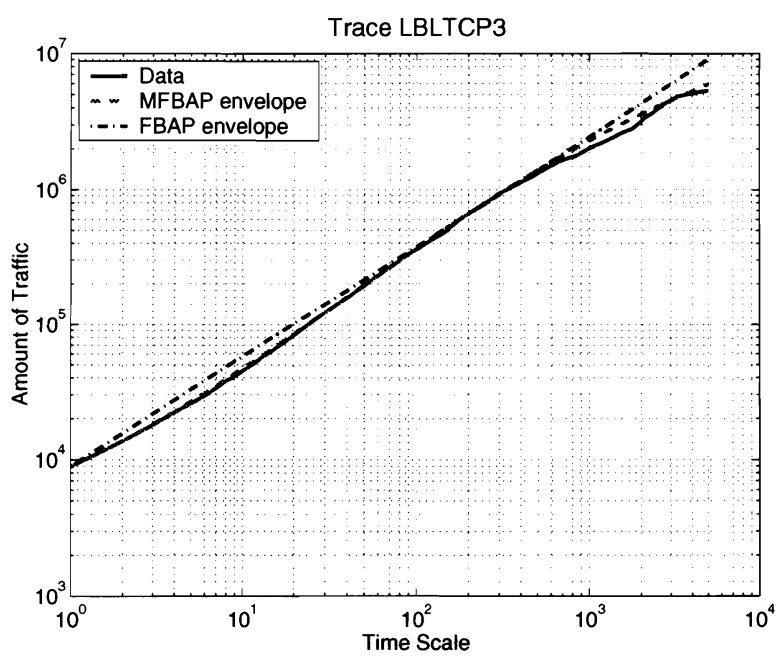

Figura 12. Relação $\log Z^{(e)}[i] \times \log i$ para o traço LBLTCP3, e sua aproximação para o caso dos modelos FBAP e MFBAP. 

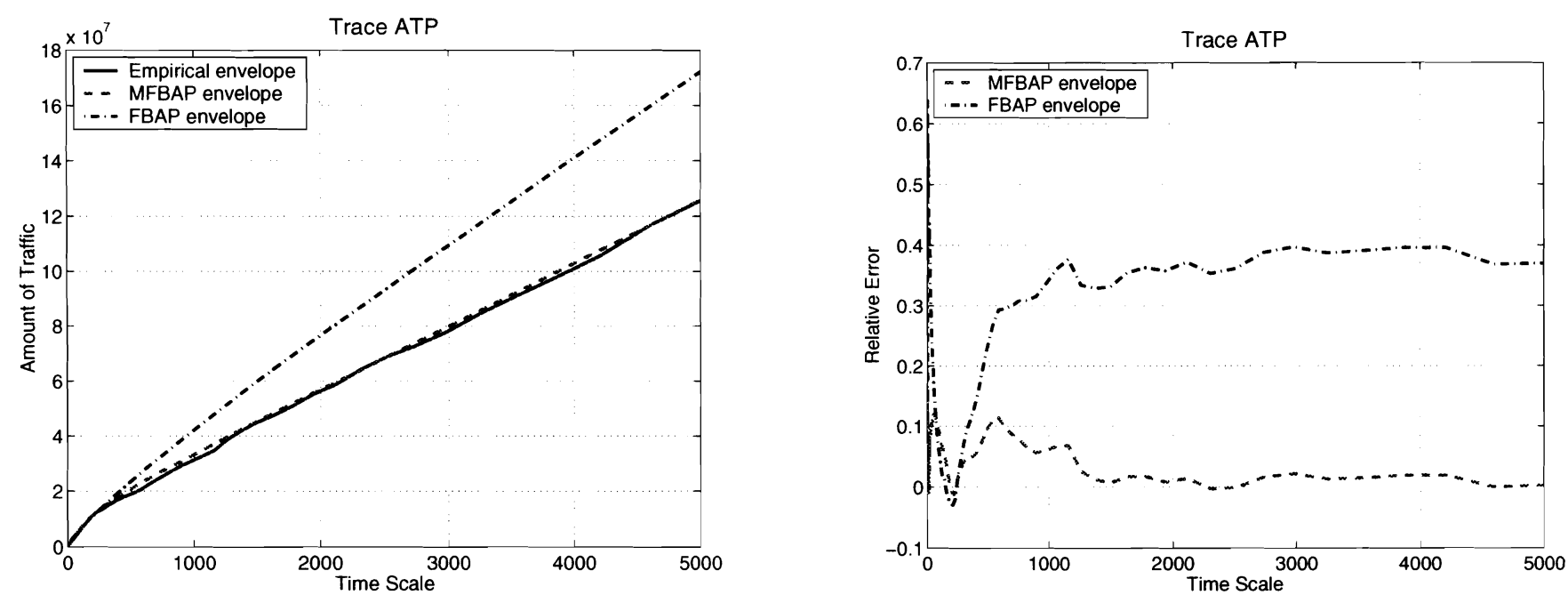

Figura 13. Representação do traço ATP utilizando os envelopes FBAP e MFBAP: (a) envelope empírico, FBAP e MFBAP; (b) erro relativo decorrente do uso dos envelopes FBAP e MFBAP.
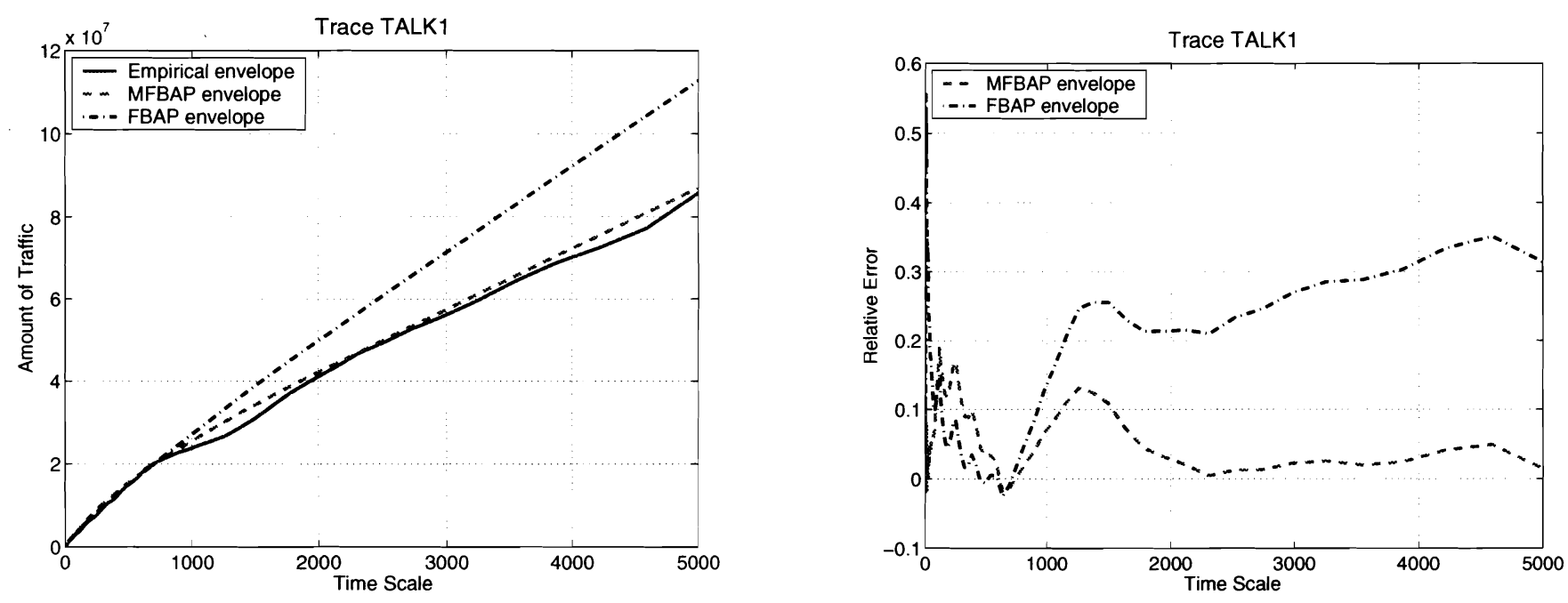

Figura 14. Representação do traço TALK 1 utilizando os envelopes FBAP e MFBAP: (a) envelope empírico, FBAP e MFBAP; (b) erro relativo decorrente do uso dos envelopes FBAP e MFBAP.
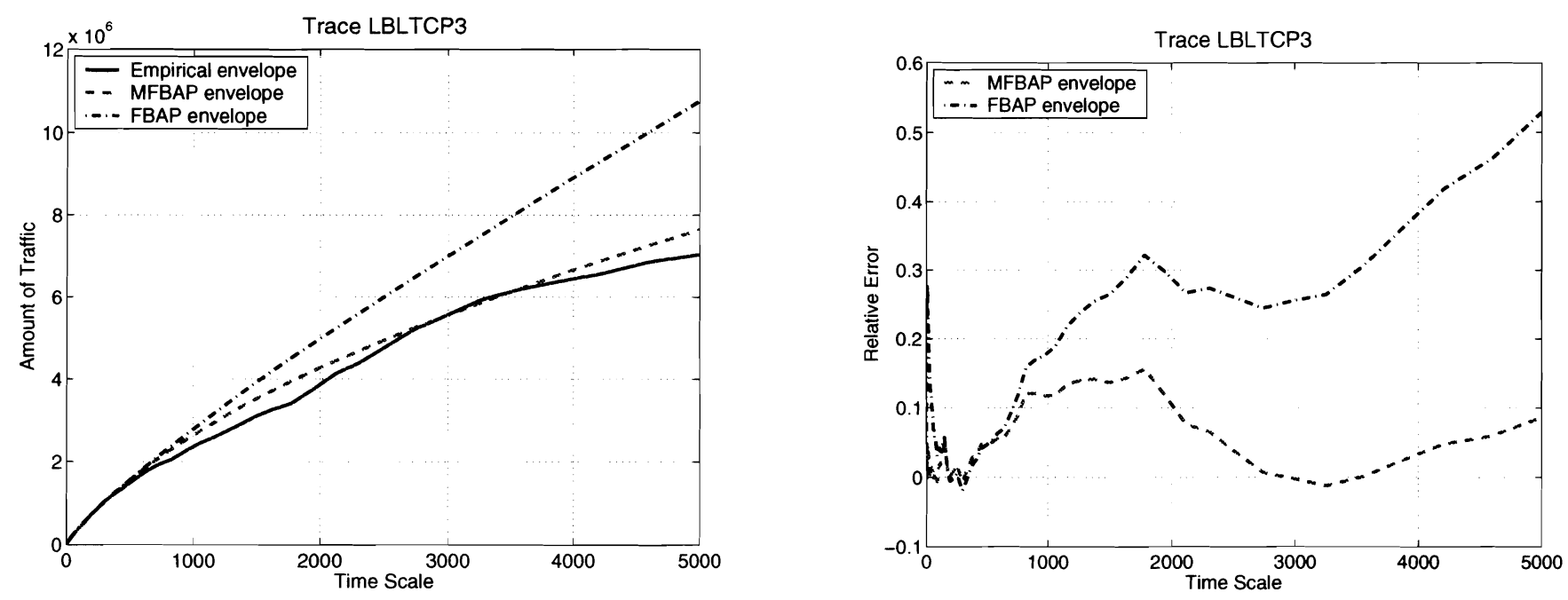

Figura 15. Representação do traço LBLTCP3 utilizando os envelopes FBAP e MFBAP: (a) envelope empírico, FBAP e MFBAP; (b) erro relativo decorrente do uso dos envelopes FBAP e MFBAP. 
Flávio de M. Pereira, Nelson L. S. da Fonseca e Dalton S. Arantes Modelagem de Tráfego Fractal Utilizando Processos Envelope

\begin{tabular}{|c|c|c|c|c|c|c|}
\hline Trace & $\mu$ & $k \gamma$ & $H_{0}$ & $\gamma_{H}$ & $\sigma_{H}$ & $\mu_{H}$ \\
\hline BCOCT89EXT & 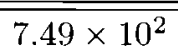 & $5.30 \times 10^{4}$ & $0 . \overline{378}$ & $\overline{0.406}$ & 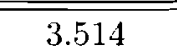 & -1.569 \\
\hline BCOCT89EXT4 & $6.22 \times 10^{3}$ & $1.86 \times 10^{5}$ & 0.489 & 0.486 & 14.681 & 0.623 \\
\hline BCPAUG89 & $1.38 \times 10^{5}$ & $4.52 \times 10^{5}$ & 0.393 & 0.374 & 11.625 & -0.428 \\
\hline ВСРОСТ 89 & $3.63 \times 10^{5}$ & $6.27 \times 10^{5}$ & 0.485 & 0.483 & 5.256 & -1.381 \\
\hline ASTERIX & $5.59 \times 10^{5}$ & $1.58 \times 10^{6}$ & 0.480 & 0.347 & 5.185 & -1.555 \\
\hline ATP & $5.47 \times 10^{5}$ & $1.14 \times 10^{6}$ & 0.468 & 0.557 & 2.130 & 0.317 \\
\hline BOND & $6.08 \times 10^{5}$ & $1.39 \times 10^{6}$ & 0.391 & 0.293 & $4.63 \times 10^{6}$ & 0.000 \\
\hline DECPKT2 & $2.38 \times 10^{5}$ & $3.85 \times 10^{5}$ & 0.419 & 0.403 & 5.955 & -1.965 \\
\hline DECPKT 3 & $1.81 \times 10^{5}$ & $3.10 \times 10^{5}$ & 0.401 & 0.403 & 6.202 & -1.630 \\
\hline DECPKT4 & $2.63 \times 10^{5}$ & $3.12 \times 10^{5}$ & 0.400 & 0.434 & 5.330 & -1.335 \\
\hline DINO & $3.27 \times 10^{5}$ & $8.48 \times 10^{5}$ & 0.491 & 0.363 & 3.204 & 0.324 \\
\hline FUSS I & $6.78 \times 10^{5}$ & $1.61 \times 10^{6}$ & 0.405 & 0.304 & 5.505 & -0.745 \\
\hline FUSS2 & $6.28 \times 10^{5}$ & $1.81 \times 10^{6}$ & 0.491 & 0.455 & 2.500 & -0.605 \\
\hline LAMBS & $1.83 \times 10^{5}$ & $7.15 \times 10^{5}$ & 0.385 & 0.497 & 3.934 & 1.759 \\
\hline LBLPKT4 & $3.64 \times 10^{4}$ & $2.58 \times 10^{5}$ & 0.409 & 0.345 & 5.472 & -2.381 \\
\hline LBLPKT 5 & $2.61 \times 10^{4}$ & $2.33 \times 10^{5}$ & 0.393 & 0.394 & 5.407 & -1.259 \\
\hline LBLTCP3 & $3.39 \times 10^{4}$ & $3.65 \times 10^{5}$ & 0.464 & 0.437 & 4.845 & -1.232 \\
\hline MOVIE & $3.57 \times 10^{5}$ & $9.93 \times 10^{5}$ & 0.392 & 0.328 & 4.778 & 0.473 \\
\hline MRBEAN & $4.41 \times 10^{5}$ & $1.39 \times 10^{6}$ & 0.543 & 0.368 & 3.214 & 0.313 \\
\hline MTV1 & $6.15 \times 10^{5}$ & $2.12 \times 10^{6}$ & 0.571 & 0.413 & 2.454 & -0.427 \\
\hline MTV2 & $4.95 \times 10^{5}$ & $2.51 \times 10^{6}$ & 0.460 & 0.372 & $5.6 \overline{25}$ & 0.476 \\
\hline NEWS 1 & $5.17 \times 10^{5}$ & $1.43 \times 10^{6}$ & 0.564 & 0.292 & 2.214 & -0.373 \\
\hline NEWS2 & $3.84 \times 10^{5}$ & $2.11 \times 10^{6}$ & 0.493 & 0.486 & $1 . \overline{932}$ & -1.278 \\
\hline RACE & $7.69 \times 10^{5}$ & $2.00 \times 10^{6}$ & 0.411 & 0.496 & 3.255 & -1.051 \\
\hline SBOWL & $5.88 \times 10^{5}$ & $1.17 \times 10^{6}$ & 0.453 & 0.429 & 3.147 & -0.159 \\
\hline SIMPSONS & $4.64 \times 10^{5}$ & $1.17 \times 10^{6}$ & 0.443 & 0.305 & 5.103 & -0.341 \\
\hline STAR2 & $2.33 \times 10^{5}$ & $7.51 \times 10^{5}$ & 0.462 & 0.465 & 3.134 & 1.546 \\
\hline TALK 1 & $3.63 \times 10^{5}$ & $6.50 \times 10^{5}$ & 0.369 & 0.608 & 3.077 & 0.870 \\
\hline TALK2 & $4.48 \times 10^{5}$ & $1.03 \times 10^{6}$ & 0.470 & 0.571 & 2.413 & 0.157 \\
\hline TERMINATOR & $2.73 \times 10^{5}$ & $5.12 \times 10^{5}$ & 0.420 & 0.281 & 4.174 & -1.038 \\
\hline
\end{tabular}

Tabela 2. Traços utilizados na validação do modelo MFBAP.

\section{CONCLUSÕES}

Neste artigo, foi proposto um modelo de tráfego autosimilar, denominado Processo de Chegada com Limitante Fracionário (Fractional Bounded Arrival Process, FBAP). A análise de filas para o tráfego FBAP foi desenvolvida, sendo estabelecidos limitantes para o máximo backlog e para o máximo retardo em um sistema de filas simples. Foi também investigado o uso do algoritmo do Balde Furado para o policiamento de tráfego FBAP. Esta abordagem é especialmente interessante no caso de redes orientadas à provisão de $\mathrm{QoS}$, dado que vários estudos de análise de desempenho de redes disponíveis na literatura assumem que o tráfego é policiado por este algoritmo. Além disso, o algoritmo do Balde Furado já tem sido utilizado em redes devido à sua robustez e baixo custo computacional.

Os resultados foram extendidos ao caso do tráfego multifractal. Um processo envelope denominado Processo de Chegada com Limitante Multifractal (Multifractal Bounded Arrival Process, MFBAP) foi proposto, sendo o modelo vali- dado utilizando traços de tráfego real. Pesquisas estão sendo conduzidas no sentido de analisar o comportamento de sistemas de filas sob tráfego MFBAP, com foco na provisão de QoS. Embora esta análise tenha sido conduzida para o caso do tráfego FBAP, a extensão dos resultados não é trivial, devido à falta de soluções analíticas para os problemas de otimização envolvidos.

\section{AGRADECIMENTOS}

Este trabalho foi financiado por FAPESP (Proc. 01/14379-4), CNPq (Proc. 300064/95-0) e Ericsson Research (Contrato UNI-35/19-2000).

\section{REFERÊNCIAS}

[1] W. E. Leland and D. V. Wilson, "High time-resolution measurement and analysis of LAN traffic: Implications for lan interconnection.," in Proc. IEEE/INFOCOM, pp. 1360-1366, apr 1991. 
[2] W. E. Leland, W. Willinger, M. S. Taqqu, and D. Wilson, "On the self-similar nature of ethernet traffic (extended version)," IEEE/ACM Trans. Networking, vol. 2, pp. 1-15, Feb. 1994.

[3] M. W. Garret and W. Willinger, "Analysis, modeling and generation of self-similar VBR video traffic," in Proc. ACM/SIGCOMM, pp. 269-280, 1994.

[4] V. Paxson and S. Floyd, "Wide area traffic: The failure of poisson modeling." IEEE Trans. Networking, vol. 3, pp. 226-244. June 1995.

[5] O. Rose, "Statistical properties of MPEG video traffic and their impact on traffic modeling in ATM systems." Tech. Rep. 101 Institute of Computer Sciences, University of Würzburg, 1995.

[6] M. Taqqu, V. Teverovsky, and W. Willinger, "Is network traffic self-similar or multifractal ?" Fractals, vol. 5, pp. 63-73, 1997.

[7] K. Park, G. Kim, and M. Crovella, "On the relation between file sizes, transport protocols and self-similar network traffic,' in Proc. IEEE Int'l. Conf. Network Protocols, pp. 171-180, Oct. 1996.

[8] O. Rose. "Simple and efficient models for variable bit rate MPEG video traffic," Perf. Eval., vol. 30, pp. 69-85, 1997.

[9] W. Willinger, M. S. Taqqu, R. Sherman, and D. V. Wilson, "Self-similarity through high-variability: statistical analysis of ethernet LAN traffic at the source level," IEEE/ACM Trans. Networking, vol. 5, pp. 71-86, Feb. 1997.

[10] M. E. Crovella, "Self-similarity in WWW traffic: evidence and possible causes," IEEE Trans. Networking, vol. 5, pp. 835845, Dec. 1997.

[11] R. H. Riedi and J. L. Véhel. "TCP traffic is multifractal: a numerical study," Research Report 3129, INRIA, Mar. 1997.

[12] A. Feldmann, A. C. Gilbert, and W. Willinger, "Data networks as cascades: Investigating the multifractal nature of internet WAN traffic," in Proc. SIGCOMM, (Vancouver, Canada), pp. 42-55, Sept. 1998.

[13] K. Park and W. Willinger, eds., Self-similar network traffic and performance evaluation. New York: Wiley, 2000.

[14] I. Norros, "On the use of fractional brownian motion in the theory of connectionless networks," IEEE J. Select. Areas Commun., vol. 13, pp. 953-962, Aug. 1995.

[15] A. Karasaridis and D. Hatzinakos, "A non-gaussian selfsimilar process for broadband heavy-traffic modeling," in Proc. IEEE/GLOBECOM, (Sydney, Australia), 1998.

[16] A. Karasaridis and D. Hatzinakos, "Broadband heavy-traffic modeling using stable self-similar processes," in 2nd Canadian Conference on Broadband Research, (Ottawa, Canada), pp. 157-168, June 1998.

[17] I. Norros, "A storage model with self-similar input," Queueing Syst., vol. 16, pp. 387-396, 1994

[18] G. S. Mayor and J. A. Silvester, "Time scale analysis of an ATM queueing system with long-range dependent traffic," in Proc. IEEE/INFOCOM, pp. 205-212, 1997.

[19] N. L. S. Fonseca, G. S. Mayor, and C. A. Viana Neto, "On the equivalent bandwidth of self-similar sources," ACM Trans. on Modeling and Computer Simulation, vol. 10, pp. 104-124, Apr. 2000

[20] T. Mikosch. S. Resnick, H. Rootzén, and A. Stegeman, "Is network traffic approximated by stable Lévy motion or fractional Brownian motion ?" Tech. Rep. 1247, Cornell University, 1999.

[21] F. M. Pereira, N. L. S. Fonseca, and D. S. Arantes, "Fractional bounded arrival process for self-similar traffic policing," in Proc. IEEE/ICC. (Anchorage, USA), May 2003.

[22] S. Molnár, T. D. Dang, and I. Maricza, "On the queue tail asymptotics for general multifractal traffic," in Proc. Networking, (Pisa, Italy), 2002.
[23] B. Mandelbrot, A. Fisher, and L. Calvet, "A multifractal model of asset returns," Cowles Foundation Discussion Paper 1164 , Yale University, Sept. 1997.

[24] P. Abry, R. Baraniuk, P. Flandrin, R. Riedi, and D. Veitch, "Multiscale nature of network traffic," IEEE Signal Processing Magazine, May 2002.

[25] R. Cruz, "A calculus for network delay, part I : Network elements in isolation," IEEE Trans. Inform. Theorv, vol. 37, pp. 114-131, Jan. 1991

[26] R. Cruz, "A calculus for network delay, part II : Network analysis," IEEE Trans. Inform. Theory, vol. 37, no. 1, pp. 132-141, 1991.

[27] M. Taqqu, V. Teverovsky, and W. Willinger, "Estimators for long-range dependence: an empirical study." Fractals, vol. 3, no. 4, pp. 785-798, 1995

[28] P. Abry and D. Veitch, "Wavelet analysis of long-range dependent traffic," IEEE Trans. Inform. Theory, vol. 44, pp. 2-15. Jan. 1998.

[29] M. Chi, E. Neal, and G. Young, "Practical applications of fractional brownian motion and noise to synthetic hydrology," Water Resources Research, vol. 9, pp. 1523-1533, Dec. 1973.

[30] "The internet traffic archive." URL: http://ita.ee.Ibl.gov/.

[31] V. Paxson, "Fast approximation of self-similar network traffic," Tech. Rep. LBL-36750, Lawrence Berkeley Laboratories. 1995.

[32] F. M. Pereira, N. L. S. Fonseca, and D. S. Arantes, "Fractal traffic modeling and policing," Relatório Técnico, 2003.

[33] G. Mayor and J. A. Silvester, "Providing QoS for long-range dependent traffic," in The 7th IEEE Computer-Aided Modeling. Analysis and Design of Communications Link and Networks, pp. 19-28, 1998.

[34] N. G. Duffield and N. O'Connell, "Large deviation and overflow probabilities for the general single-server queue, with applications," Proc. Cambridge Philosophical Society, no. 118, pp. 363-374, 1995.

Flávio de Melo Pereira nasceu no Rio de Janeiro, Brasil, em 1976. Em 1998, formou-se em Engenharia Elétrica pela Universidade Estadual de Campinas. Entre 1999 e 2000, trabalhou como engenheiro de software na Alcatel Telecomunicações S/A. Em 2002, obteve o título de Mestre em Engenharia Elétrica, modalidade Telecomunicações e Telemática, pela Unicamp. Desde 2001, é aluno do curso de Doutorado em Engenharia Elétrica da Unicamp, modalidade Telecomunicações e Telemática. Atualmente, ocupa o cargo de analista de supervisão bancária do Banco Central do Brasil.

Nelson Luis Saldanha da Fonseca recebeu o grau de Engenheiro Eletricista e Mestre em Informática pela Pontifícia Universidade Católica do Rio de Janeiro e os graus de M.Sc. e Ph.D. em Computer Engineering pela University of Southern California. Desde 1995 pertence ao corpo docente do Instituto de Computação da Universidade Estadual de Campinas, onde ocupa o cargo de Professor Associado. Publicou mais de 100 artigos e capítulos de livros. Nelson Fonseca é editor da e-news da ComSoc and ViceChair de dois comitês técnicos: Multimedia Communications and Communications Systems Integration and Modeling. Foi Director of On-line Services (2002-2003) e Editor-in-Chief of JEEE Global Communications Newsletter (1999-2002). Atualmente é membro do corpo editorial da Revista Brasileira de Telecomunicações, IEEE Transactions on Multimedia, IEEE Communications Magazine, IEEE Communications Surveys and Tutorials e Computer Networks. Nelson Fonseca foi co-chair das seguintes eventos: General Symposium (Globecom 2004), QoS and Performance Modeling Symposium (ICC2004), Communication QoS, Reliability and Performance Modeling Symposium (ICC'03), International Teletraffic 
Flávio de M. Pereira, Nelson L. S. da Fonseca e Dalton S. Arantes

Modelagem de Tráfego Fractal Utilizando Processos Envelope

Congress'01 e Multimedia Technology and Services Symposium (Globecom'99).

Dalton Soares Arantes recebeu o grau de Engenheiro Eletrônico pela Universidade Federal do Rio de Janeiro em 1969, o grau de Mestre em Engenharia Elétrica pela Universidade Estadual de Campinas em 1972, e o grau de Ph.D. pela Cornell University em 1976. Entre 1993 e 1995, foi professor visitante no Depto. de Engenharia Elétrica e de Engenharia de Computação da Universidade do Novo México, EUA. Atualmente, é professor de Engenharia Elétrica na Unicamp, onde já ocupou os cargos de Chefe do Depto. de Engenharia Elétrica e de Chefe do Depto. de Comunicações. Entre 1976 e 1999, coordenou diversos projetos de pesquisa em comunicação digital, em cooperação com o CPqD. Suas pesquisas incluem trabaIhos nas áreas de processamento adaptativo de sinais, comunicação móvel, redes de computadores, modelagem de tráfego e simulação. 